\title{
MODELO OBJETO-REQUERIMIENTO-ACTIVIDAD PARA MODELAR FLUJOS DE TRABAJO PARA LA GESTIÓN DE REQUERIMIENTOS DE DESARROLLO DEL PRODUCTO EN LA INDUSTRIA DE LA CONFECCIÓN
}

\author{
Object-Request-Activity model for modeling workflows of requests for \\ product development in the garment industry
}

\author{
Juan Sotelo Villena, Javier Sánchez Espinoza'
}

\section{RESUMEN}

En este artículo se formula un modelo general que actúa como plantilla o marco de referencia para facilitar la concepción de los flujos de trabajo particulares en cada empresa. El modelo denominado ORA generaliza el diseño de flujos de trabajo de las áreas de Desarrollo del Producto (DDP) de la industria de confecciones textiles. Así mismo, se propone el diseño de un sistema de seguimiento de requerimientos para el área de DDP, basado en el modelo genérico de flujo de trabajo propuesto. Además, se logra identificar los principales requerimientos de DDP del sector de la industria de la confección y se demuestra que, aplicando el modelo ORA, se pueden modelar todos los flujos de trabajo necesarios para gestionar eficazmente los requerimientos de las áreas de DDP de este sector industrial.

Palabras clave: Modelado de flujos de trabajo, tecnología workflow, Business Process Management (BPM), requerimientos de desarrollo del producto, gestión por procesos, workflow management system.

\begin{abstract}
This paper presents a general model, which acts as a template or framework to facilitate the design of individual flows for particular business. The model called ORA, generalizes the workflow design of the Development Product areas (DDP) in textile manufacturing industry. Also the design of a system for monitoring requirements is presented based on the model ORA. The main requirements of DDP in garment industry are identified and it is shown that using the ORA model can model all the workflows required to effectively manage requirements in DDP areas of this industry.

Keywords: workflow modeling, workflow technology, Business Process Management (BPM) requirements for product development, process management, workflow management system
\end{abstract}




\section{INTRODUCCIÓN}

La globalización de la economía ha creado un escenario de mercado que se caracteriza, principalmente, por clientes más exigentes y mejor informados, la individualización de los mismos, plazos menores de entrega y márgenes más ajustados. Esta situación ha obligado a las empresas a cuestionar sus enfoques de gestión. En este contexto, ha surgido una serie de nuevas corrientes de gestión como ISO y EFQM, que promueven un enfoque de gestión por procesos de negocio (Beltran, 2004 y Ogalla, 2006) que tienen como premisa que son los procesos los que generan productos y servicios para el cliente, y que lo realmente importante es satisfacer al cliente en cuanto a calidad, precio y plazo. De esa manera, se obtendrá un margen razonable.

La industria de la confección textil del Perú es principalmente exportadora y altamente sensible al contexto descrito. Las empresas de este sector fabrican bajo pedido. Es decir, reciben especificaciones de diseño, las desarrollan y, si se satisface al cliente con la aprobación tanto del desarrollo como del precio cotizado, se reciben los pedidos y se procede a manufacturarlos para despacharlos finalmente. Debido a la alta variedad y rotación de la moda, las áreas de DDP de las empresas de este sector reciben innumerables requerimientos (cientos o miles) para el desarrollo de prendas de vestir. Gracias a los acuerdos de comercio exterior como el ATPDEA, primero, y luego los TLC firmados con USA y otros países, los volúmenes de exportación se han incrementado de manera notable, a pesar de los baches creados por las crisis internacionales (ver figura 1). Esta situación ha incrementado de manera considerable la cantidad de requerimientos de DDP que deben resolverse y ha creado un problema de gestión en esta área.

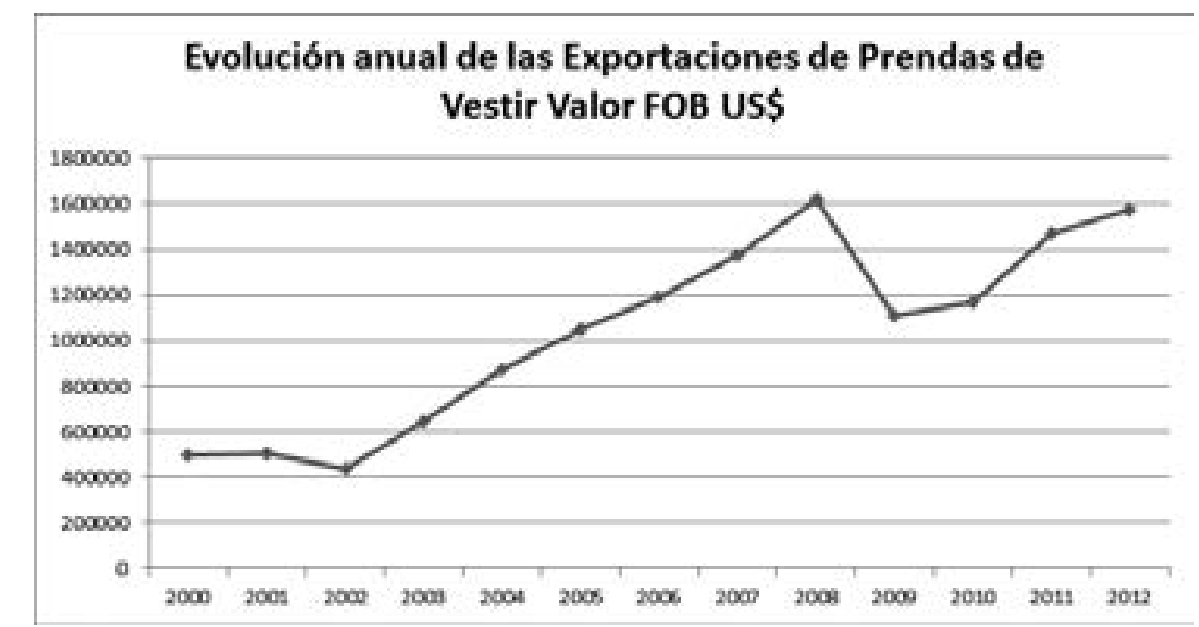

Fig. 1. Evolución de las exportaciones peruanas de prendas de vestir. Fuente: (Sociedad Nacional de Industrias, 2013).

En general, para soportar la gestión por procesos, claramente necesaria para el caso descrito, las TIC han incorporado tecnologías basadas en flujos de trabajo. Según Muehlen (2004), estos pueden definirse como la representación específica de un proceso que está diseñado de forma que los mecanismos de coordinación formal entre actividades, las aplicaciones y los participantes del proceso pueden ser controlados por un sistema de información llamado Workflow Management System (WMS).

Aparentemente, el problema estaría resuelto. Sin embargo, las herramientas de software de flujo de trabajo exigen que cada empresa, primero, identifique todos los tipos de requerimientos y, luego, modele los procesos para atenderlos. Ello plantea un problema de abstracción y modelado que, 
generalmente, no toman en cuenta ni los responsables del proceso, ni los informáticos que proveen soluciones de flujo de trabajo. La identificación de esta brecha originó el espacio de investigación para el presente estudio.

\section{PLANTEAMIENTO DEL PROBLEMA}

Las áreas de DDP de la industria de confección textil, debido a la moda, manejan miles de requerimientos de desarrollo y generalmente no logran controlar de manera eficaz el seguimiento de los procesos. Es muy común que, por tratarse de actividades netamente técnicas y especializadas, los responsables de DDP sean personas altamente calificadas en los aspectos técnicos de las actividades que se realizan, pero no necesariamente modeladores de los procesos que realizan. Además, los informáticos que proveen y soportan herramientas de flujo de trabajo suelen tener poco conocimiento de los procesos de negocio de una industria en particular. Una semblanza del ciclo de negocio de la industria de la confección textil y las principales actividades de DDP se puede ver a continuación (figura 2).

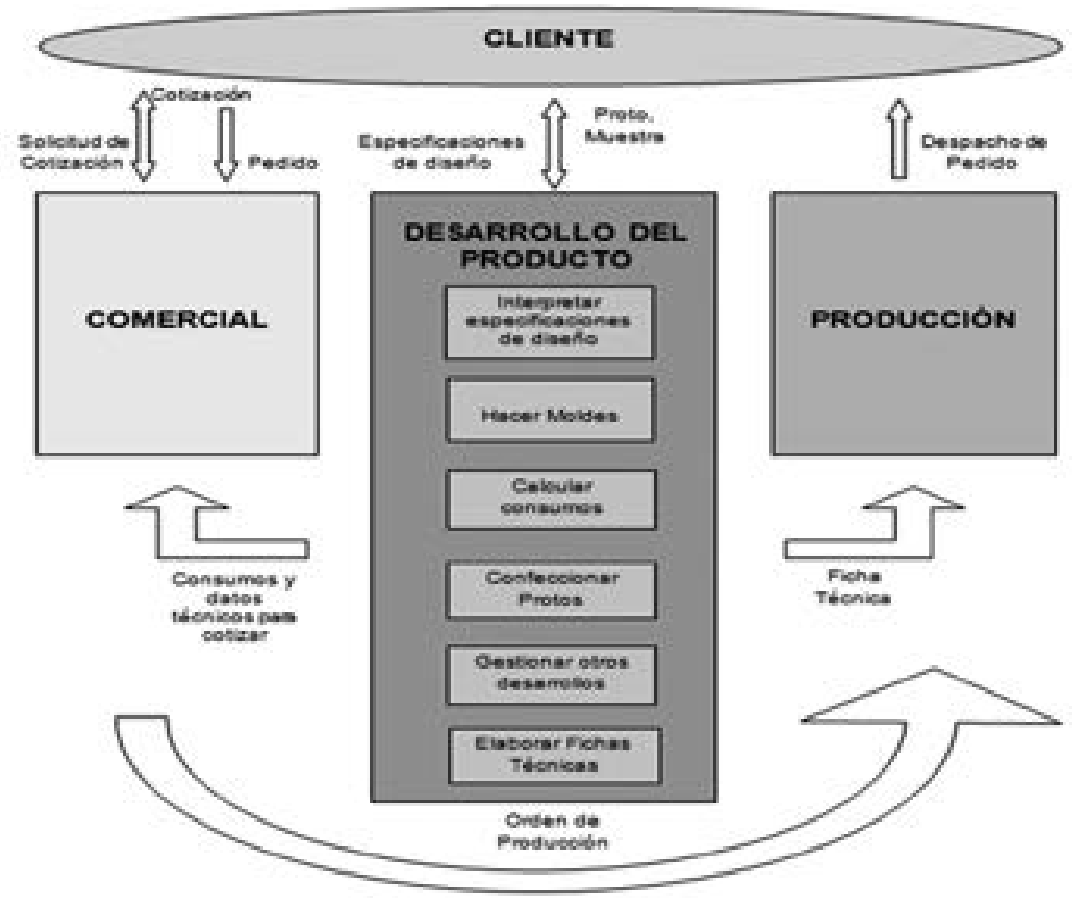

Fig. 2. Ciclo de negocio de la industria de la confección textil.

Las preguntas típicas que no tienen respuesta oportuna son las siguientes:

- ¿Cuántos requerimientos pendientes hay en proceso?

- ¿Qué nivel de atraso tienen?

- ¿En qué parte del proceso esta cada uno?

- ¿Qué actividad es el cuello de botella del proceso?

Entre los síntomas más notorios del problema se presentan los siguientes:

- Retraso en la atención de requerimientos del cliente, 
- Falta de visibilidad del proceso, ya que no se conoce exactamente el estado de cada requerimiento (falta de información oportuna),

- Demasiado tiempo para resolver los requerimientos de desarrollo (protos, muestras, desarrollos de tela, color, etc.),

- Envíos repetitivos de protos, por comentarios desfavorables del cliente,

- Alteración permanente de las prioridades en la atención de los requerimientos.

La respuesta natural a la situación problema es aplicar algún esquema de gestión por proceso que permita detectar oportunamente los cuellos de botella y aplicar mejoras de procesos. Además, la tecnología de flujo de trabajo soporta este tipo de gestión. Entonces, el problema a resolver es ¡cómo facilitar la tipificación y modelado de flujos de trabajo para la industria de confección textil para gestionar requerimientos de DDP?

\section{MARCO CONCEPTUAL}

Seguidamente, se definen algunos conceptos importantes aplicados en el estudio.

Proceso de negocio: secuencia lógica, discreta, holística y, temporal de actividades que son necesarias para manipular un objeto relevante económicamente (Muehlen, 2004).

Business Process Management (BPM): conjunto de métodos, herramientas y tecnologías utilizados para diseńar, representar, analizar y controlar procesos de negocio operacionales (Garimella y Williams, 2009). Los sistemas BPM están guiados por modelos explícitos que representan la lógica de los procesos de negocio, que es abstraída de las aplicaciones que los ejecutan (Borja, 2004).

Flujo de trabajo (Workflow): automatización de un proceso de negocio total o parcial, en el que documentos, información o tareas son pasados de un usuario a otro para que tome acción de acuerdo a un conjunto de reglas (WfMC, 1999). La figura 3 muestra el esquema básico de un flujo de trabajo.

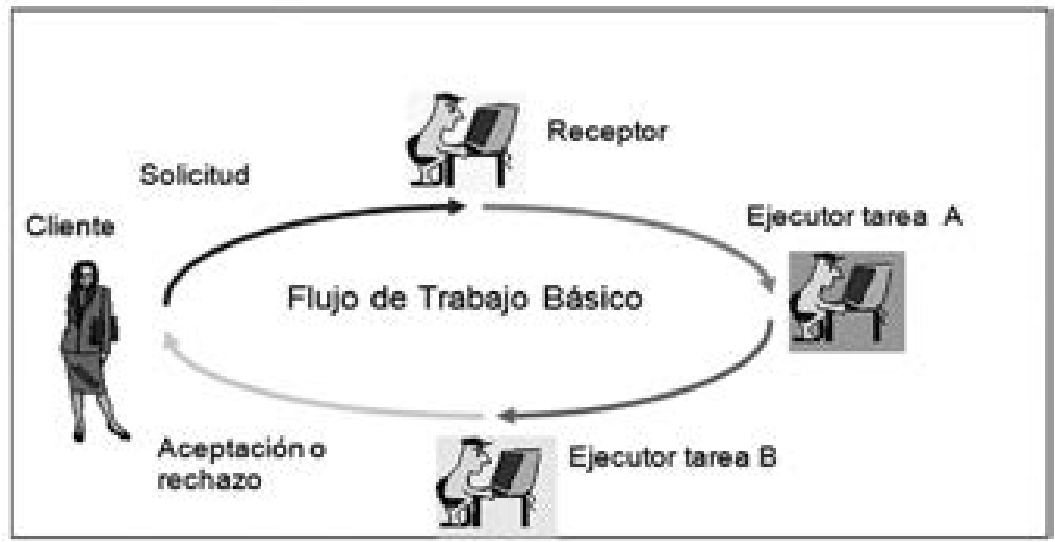

Fig. 3. Esquema básico de un flujo de trabajo.

Workflow Management Systems (WMS): sistema que define, crea y gestiona la ejecución de flujos de trabajo mediante el uso de software. De esa manera, es capaz de interpretar la definición del proceso, interactuar con los participantes y, siempre que se requiera, invocar el uso de herramientas y aplicaciones (WfMC, 1999). Un WMS puede ser comparable con un sistema de administración de bases de datos. Es un sistema genérico que opera sobre un esquema de definición (Petkov y Haller, 2005). 
Tanto Penadés (2002) y Muehlen (2004), coinciden en afirmar que, de la definición de WMS ofrecida por la WfMC (1999), se desprende que, en un WMS, existen dos grandes funciones claramente delimitadas e interactuantes. Por una parte, se debe considerar la que corresponde a la creación de los modelos de flujo de trabajo. Por otro lado, existe la que es propia de la creación y ejecución de las instancias de los modelos de flujo de trabajo. La figura 4 presenta cómo está compuesto funcionalmente un WMS.

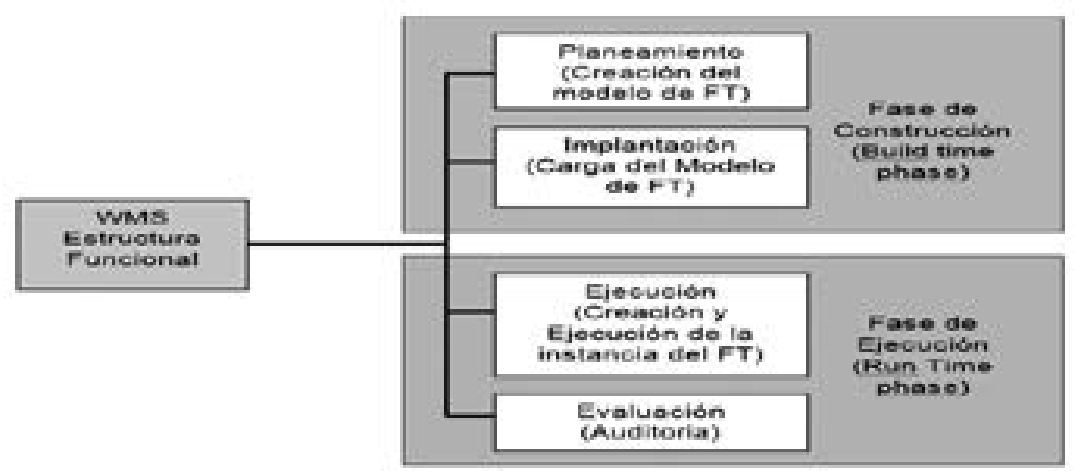

Fig. 4. Descomposición funcional de un WMS. Fuente: (Mueblen, 2004).

\section{METODOLOGÍA DEL ESTUDIO}

La investigación que se presenta utilizó el esquema de la investigación basada en el caso. Según Mertens, Grinnel y otros autores citados por Hernández (2006), los estudios del caso pueden definirse, al aplicar los procesos de la investigación cuantitativa, cualitativa o mixta, como estudios de la unidad de análisis u objeto para responder al planteamiento del problema, probar alguna hipótesis o desarrollar alguna generalización.

Para desarrollar el modelo, se aplicó el método inductivo que, según Bernal (2006), parte del estudio de los hechos particulares aceptados como válidos para llegar a conclusiones de aplicación general. En la investigación, se buscó la generalización a partir de las experiencias particulares en diferentes empresas de un mismo sector industrial.

En referencia a la data requerida para la investigación, la población estuvo conformada por los requerimientos de DDP que reciben todas las empresas del sector de la industria de la confección, preferentemente las exportadoras. La muestra estuvo representada por los requerimientos de un grupo de empresas en las que se han tenido experiencias de identificación y tratamiento del problema. El área de DDP de cualquier empresa de la industria de la confección constituyó el objeto de estudio o unidad de análisis.

Se aplicó el diseño de investigación inductivo, dado que necesariamente se tuvo que partir por la identificación y análisis de los requerimientos particulares, para descubrir similitudes y propiedades comunes, y lograr la abstracción general que represente adecuadamente a cada caso en particular. Se desarrolló un método de cuatro pasos para la generación del modelo ORA.

\section{Paso 1: Identificar y analizar los requerimientos}

Busca realizar un levantamiento de información (trabajo de campo) para detectar las diferentes solicitudes que reciben las áreas de DDP de parte de los diferentes clientes. Toma en cuenta el 50\% de la data recolectada para realizar el análisis y deja el otro 50\% para el proceso de validación. El análisis 
de cada requerimiento se realiza desde la óptica de la gestión del proceso. De esa manera, se detecta cuáles son las tareas claves que se deben completar para atender un requerimiento.

\section{Paso 2: Abstraer los elementos conceptuales}

A partir del análisis de la muestra, debe realizarse un proceso de abstracción para identificar los elementos conceptuales: los objetos de negocio, los requerimientos que ellos generan, las actividades que se deben controlar para mantener un seguimiento de la atención de los requerimientos y los usuarios involucrados. En este paso, es importante verificar que los elementos conceptuales sean comunes a todo tipo de requerimiento, independientemente del cliente que lo solicita o de la fábrica que lo atiende.

\section{Paso 3: Estructurar el Modelo a partir de los elementos conceptuales}

Se organiza la interacción de todos los elementos conceptuales abstraídos en el paso anterior. En consecuencia, se propone la estructura conceptual del modelo y se verifica que cualquier caso de la muestra analizada pueda ser representable por el modelo generado.

\section{Paso 4: Validar el Modelo}

Se validan la viabilidad (capacidad de instrumentarse) y la generalización (capacidad de modelar cualquier requerimiento de DDP). Para el proceso de validación se plantean 2 pasos:

Primero: Diseñar y construir un sistema software basado en el modelo ORA para evidenciar que es posible instrumentar el seguimiento de requerimientos independientemente de los clientes que solicitan los requerimientos e independientemente de las empresas que los atienden.

Segundo: Modelar los flujos de trabajo del 100\% de los requerimientos que conforman la muestra aplicando el sistema software que implementa el Modelo ORA, para comprobar la generalización del modelo.

\section{DESARROLLO DEL MODELO ORA}

Para identificar la muestra de requerimientos se realizó una serie de entrevistas a gerentes comerciales y de DDP. Se identificaron 68 requerimientos de diferentes clientes, que fueron atendidos por diferentes fábricas. Por confidencialidad, se mantienen en reserva. Los requerimientos correspondieron a seis clientes conocidos en el mercado global de las prendas de vestir y accesorios, como Polo Ralph, Lands End, LL Bean, Guess, Dillar's y Eddy Bauer. La tabla 1 muestra la lista de requerimientos identificados.

Se tomó aleatoriamente el 50\% de la muestra para el análisis. Por cada requerimiento, se identificó el objeto de negocio generado, la lista de actividades o hitos necesarios para completar la atención del requerimiento, el responsable de cada actividad o hito, y se anotaron algunas observaciones o condiciones. La tabla 2 muestra el parte del resultado del análisis para los requerimientos de dos clientes. El análisis encontró lo siguiente:

1. Requerimientos iguales solicitados por diferentes clientes,

2. Requerimientos iguales, pero con diferente denominación dada por el cliente. Ejemplo: desarrollo de tela y Knitdown,

3. Requerimientos diferentes, pero con la misma lista de actividades a controlar. Ejemplo: Proto Fit Sample, Proto Size Set, Proto PP Sample,

4. En casi todos los requerimientos, es necesario el comentario del cliente. 


\begin{tabular}{lll}
\hline Cliente & Nro. & \multicolumn{1}{c}{ Requerimiento } \\
\hline & 1 & Desarrollo de Tela \\
\hline 2 & Desarrollo de Color \\
\hline 3 & Cotización \\
\hline 4 & Designer Sample \\
\hline 5 & Fit Sample \\
\hline 6 & Pre-Production Sample \\
\hline 7 & Muestra de Vendedor \\
\hline 8 & Brand Sample \\
\hline 9 & Molde de Producción \\
\hline 10 & Aprobación Tono y Matching \\
\hline 11 & Aprobación Hilo de Coser \\
\hline 12 & Testing Tela \\
\hline 13 & Testing Prenda \\
\hline 14 & Cotización \\
\hline 15 & Knitdown \\
\hline 16 & Desarrollo de Color \\
\hline 17 & Presentation Fabric \\
\hline 18 & Counter Sample \\
\hline 19 & Size Set \\
\hline 20 & Pre-Production Sample \\
\hline 21 & Muestra de Vendedor \\
\hline 22 & Molde de Producción \\
\hline 23 & Bulk Fabric \\
\hline 24 & In Line Sample \\
\hline & \\
\hline
\end{tabular}

Tabla 1. Lista de requerimientos (muestra).

6.

\begin{tabular}{|c|c|c|c|}
\hline \multicolumn{4}{|c|}{ Cliente LL Bean } \\
\hline Requerimiento & Objeto que lo genera & Actividades a realizar & Responsable \\
\hline \multirow[t]{6}{*}{ Muestra de vendedor } & Estilo & Emitido por & DDP \\
\hline & & Molde es elaborado por & Moldaje \\
\hline & & Tela producida por & Muestra telas \\
\hline & & Muestra producida por & Taller de Protos \\
\hline & & Enviada al cliente por & Ventas \\
\hline & & Comentado por & Cliente \\
\hline \multirow[t]{3}{*}{ Swatch Card } & Tela-color & Emitido por & DDP \\
\hline & & Enviado al cliente por & Testing \\
\hline & & Comentado por & Cliente \\
\hline \multicolumn{4}{|c|}{ Cliente Polo Ralph } \\
\hline \multirow[t]{4}{*}{ Desarrollo de Color } & Color & Emitido por & DDP \\
\hline & & Producido por & Laboratorio \\
\hline & & Envido al cliente por & DDP \\
\hline & & Comentado por & Cliente \\
\hline
\end{tabular}




\begin{tabular}{clll}
\hline \multirow{2}{*}{ Proto Size Set } & \multirow{2}{*}{ Estilo } & Emitido por & DDP \\
\cline { 3 - 4 } & & Molde elaborado por & Moldaje \\
\cline { 3 - 4 } & & Proto producido por & Taller de Protos \\
\cline { 3 - 4 } & & Enviado al cliente por & DDP \\
\cline { 3 - 4 } & & Comentado por & Cliente \\
\hline \multirow{2}{n}{ Run Card } & Orden de Teñido & Emitido por & DDP \\
\cline { 2 - 4 } & & Enviado al cliente por & Testing \\
\cline { 3 - 4 } & & Comentado por & Cliente \\
\hline
\end{tabular}

Tabla 2. Análisis de requerimientos.

Estas observaciones motivaron la necesidad de realizar una agrupación de requerimientos con la misma secuencia de actividades. Los 34 requerimientos analizados se agruparon en seis grupos (tabla 3), lo cual indicó que solo había el mismo número de secuencias diferentes de actividades para resolver cualquier requerimiento.

\begin{tabular}{|c|c|c|c|c|}
\hline Grupo & Secuencia de actividades & Requerimiento & Objeto de negocio & Cliente \\
\hline \multirow{6}{*}{1 PROTO } & \multirow{6}{*}{$\begin{array}{c}\text { Emitir } \\
\text { Elaborar Molde } \\
\text { Elaborar Proto } \\
\text { Enviar al cliente } \\
\text { Recibir comentario del Cliente }\end{array}$} & $\begin{array}{c}\text { Fit Sample } \\
\text { Confirmation Sample } \\
\text { PP Sample }\end{array}$ & Estilo & LL Bean \\
\hline & & $\begin{array}{l}\text { Style Sample } \\
\text { Fit Sample } \\
\text { Mock up } \\
\text { Confirmation Sample }\end{array}$ & Estilo & Lands End \\
\hline & & Size Set & Estilo & Polo Ralph \\
\hline & & $\begin{array}{l}\text { Presentation fabric } \\
\text { Counter Sample }\end{array}$ & Estilo & Eddy Bauer \\
\hline & & $\begin{array}{l}\text { Designer Sample } \\
\text { Fit Sample } \\
\text { Brand Sample }\end{array}$ & Estilo & Dillar's \\
\hline & & Size Set & Estilo & Guess \\
\hline \multirow{4}{*}{2 COTIZACIÓN } & \multirow{4}{*}{$\begin{array}{c}\text { Emitir } \\
\text { Elaborar Molde } \\
\text { Calcular consumos } \\
\text { Cotizar } \\
\text { Enviar al Cliente } \\
\text { Recibir Comentario del Cliente }\end{array}$} & Cotización & Estilo & Lands End \\
\hline & & Cotización & Estilo & Polo Ralph \\
\hline & & Cotización & Estilo & Dillar's \\
\hline & & Cotización & Estilo & LL Bean \\
\hline $\begin{array}{c}3 \text { MOLDE } \\
\text { DEPRODUCCIÓN }\end{array}$ & $\begin{array}{c}\text { Emitir } \\
\text { Elaborar Molde }\end{array}$ & Molde de Producción & Estilo & Lands End \\
\hline \multirow[b]{2}{*}{$\begin{array}{c}4 \text { MUESTRA DE } \\
\text { VENTA }\end{array}$} & Emitir & Sale Sample & Estilo & Guess \\
\hline & $\begin{array}{c}\text { Elaborar Molde } \\
\text { Fabricar Tela } \\
\text { Elaborar Proto } \\
\text { Enviar al Cliente } \\
\text { Recibir Comentario del Cliente }\end{array}$ & Muestra de Vendedor & Estilo & LL Bean \\
\hline
\end{tabular}




\begin{tabular}{|c|c|c|c|c|}
\hline \multirow{6}{*}{$\begin{array}{l}5 \text { DESARROLLO } \\
\text { TELA/COLOR }\end{array}$} & \multirow{6}{*}{$\begin{array}{c}\text { Emitir } \\
\text { Elaborar Molde } \\
\text { Fabricar Tela } \\
\text { Elaborar Proto } \\
\text { Enviar al Cliente } \\
\text { Recibir Comentario del Cliente }\end{array}$} & Desarrollo de Tela & Estilo & LL Bean \\
\hline & & Knitdown & Estilo & Lands End \\
\hline & & Desarrollo de Color & Estilo & Polo Ralph \\
\hline & & Knitdown & Estilo & Eddy Bauer \\
\hline & & Desarrollo de Color & Estilo & Dillar's \\
\hline & & Desarrollo de Tela & Estilo & Guess \\
\hline \multirow{7}{*}{6 TESTING } & \multirow{7}{*}{$\begin{array}{c}\text { Enviar al Cliente } \\
\text { Recibir Comentario del Cliente }\end{array}$} & Trim Card & Estilo & \multirow{2}{*}{ LL Bean } \\
\hline & & Swatch Card & Tela-Color & \\
\hline & & In Line Sample & Estilo & \multirow{3}{*}{ Polo Ralph } \\
\hline & & Run Card & Orden de Teñido & \\
\hline & & Bulk Fabric & Tela-Color & \\
\hline & & Shipment Sample & Estilo & Eddy Bauer \\
\hline & & Testing Tela & Pedido & Dillar's \\
\hline
\end{tabular}

\section{Tabla 3. Agrupación de requerimientos}

Además, se encontró que solo cuatro objetos de negocio generaban los diferentes requerimientos. La interrelación obtenida, por su parte, permitió generar la estructura base del modelo ORA, que se muestra en la figura 5 .

De la estructura conceptual se desprenden tres definiciones:

El Objeto de Negocio es el elemento que origina el requerimiento, por ejemplo, estilo, tela, pedido, etc. Un objeto de negocio pertenece a un cliente. También, puede generar uno o más requerimientos. Por ejemplo, un estilo puede originar los siguientes requerimientos: cotización, Proto Fit Sample, Proto Size Set, Top Sample, etc.

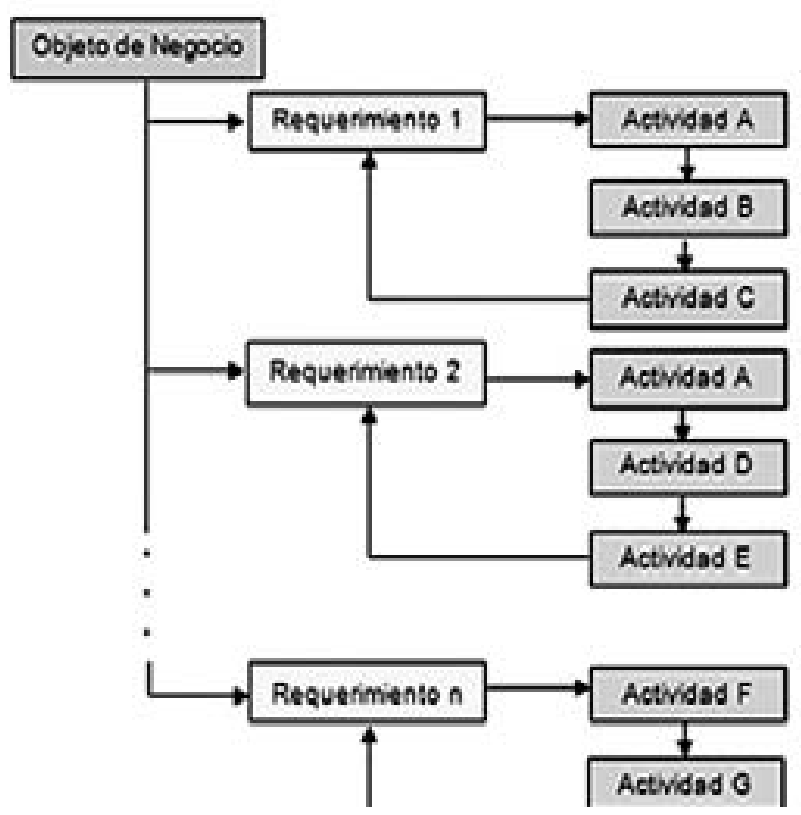

Fig. 5. Estructura conceptual del modelo ORA. 
El Requerimiento es una solitud que realiza un cliente para un objeto de negocio, por ejemplo, un Proto Fit simple.

Con respecto a la Secuencia de Actividades, todo requerimiento debe ser resuelto mediante ella. Cada actividad tiene asignado un tiempo límite para ser concluida (lead time). Un flujo de trabajo está formado por la secuencia de actividades que se deben realizar para atender el requerimiento de un cliente.

La figura 6 muestra un ejemplo de aplicación de la estructura conceptual del modelo ORA.

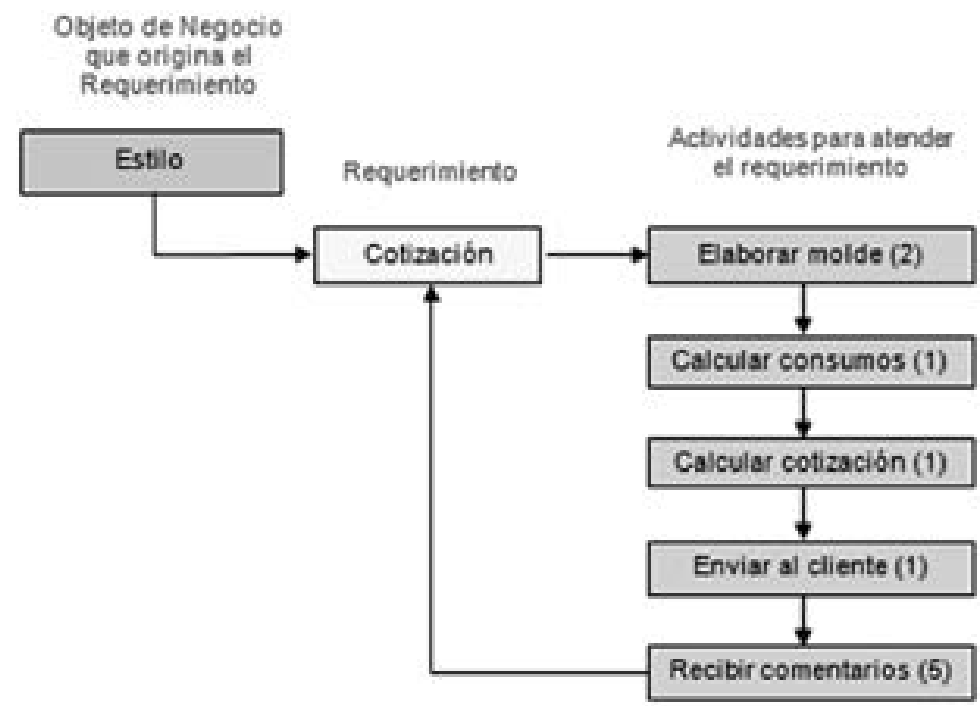

Fig. 6. Aplicación de la estructura del modelo ORA a un requerimiento especifico.

Luego de formular la estructura estática, se derivó la estructura dinámica para completar el modelo ORA. El análisis de los requerimientos de la muestra determinó que son los clientes los que solicitan los diversos requerimientos. También, se encontró que se formaban naturalmente los grupos de requerimientos con la misma secuencia de actividades. Esto permitió incluir, como elementos del modelo, tanto al cliente como al grupo de requerimiento. De esta manera, fue posible plantear que cada grupo de requerimiento tiene una secuencia de actividades asociada y que cualquier requerimiento que pertenece a cada grupo toma como suya la secuencia de actividades que corresponde. Si se entiende por «clase» la definición dada por Booch, Rumbaugh y Jacobson (2006), un grupo de requerimiento es una clase y un requerimiento es una instancia que hereda la secuencia de actividades de la clase a la que pertenece.

Asimismo, se determinó que toda actividad de una secuencia de actividades es ejecutada o es responsabilidad de algún usuario. Además, la atención o supervisión de los requerimientos de un cliente es responsabilidad de algún usuario. Por lo tanto, se propuso al usuario como un elemento de soporte del modelo ORA. Sobre este último punto se debería añadir que, según Sotelo (2007), los sistemas basados en flujo de trabajo son, por definición, proactivos. Es decir, comunican el estado de los requerimientos automáticamente a los usuarios asignados para tal propósito.

Sobre la base de la integración de las estructuras estática y dinámica del modelo, se plantearon las definiciones del modelo ORA.

1. Cliente: elemento que genera los objetos de negocio, los cuales generan requerimientos. 
2. Actividad: conjunto de tareas que deben ejecutarse para indicar un avance en la atención de un requerimiento.

3. Objeto de negocio: elemento que genera requerimientos (solicitudes de desarrollo). Pertenece a un cliente.

4. Usuario: persona que es responsable de ejecutar una actividad o de la atención de un cliente.

5. Secuencia de actividades: lista ordenada de actividades que deben ejecutarse para dar por terminada la atención de un requerimiento.

6. Grupo de requerimiento: denominación dada a una cierta secuencia de actividades.

7. Tipo de requerimiento: denominación dada a una secuencia de actividades con plazos de tiempo (Lead Time) asignados a cada actividad. Un tipo de requerimiento es un patrón que está asociado a un grupo de requerimiento, del cual hereda la secuencia de actividades.

8. Requerimiento: denominación dada a una instancia del patrón tipo de requerimiento. Todo requerimiento hereda, del tipo de requerimiento, la secuencia de actividades con plazos de tiempo asignados. El requerimiento está asociado a un objeto de negocio.

\section{VALIDACIÓN DEL MODELO}

La validación del modelo se planteó en dos frentes: primero, en demostrar la viabilidad del modelo ORA mediante un prototipo de software y, segundo, en modelar, con el prototipo, todos los requerimientos identificados en la muestra.

Arquitectura del sistema: Se planteó una arquitectura con seis componentes: base de datos, modelador de flujos de trabajo, motor de flujo de trabajo, activador de correo, generador de reportes e integrador (figura 7).

La arquitectura considera dos tipos de usuarios. En primer lugar, toma en cuenta al táctico, encargado del modelado y del monitoreo de avance de las actividades del flujo. En segundo lugar, se considera al operativo, encargado de emitir los requerimientos y de realizar las actividades del flujo.

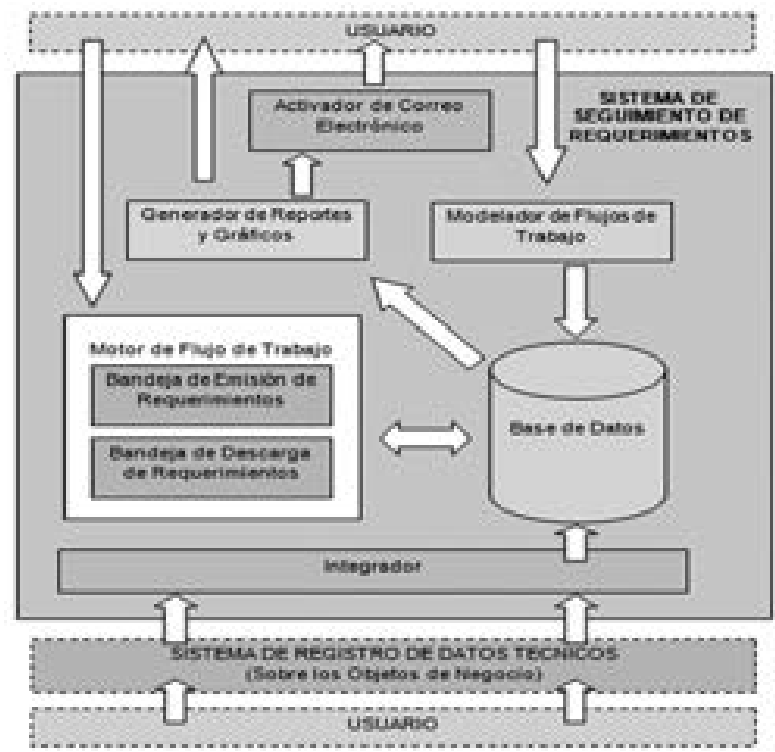

Fig. 7. Arquitectura del sistema para implementar el modelo ORA. 
Casos de uso: definen la funcionalidad del sistema. Se identificaron 16 casos de uso para el prototipo, los cuales determinan todas las interacciones que los 2 tipos de actores, identificados en la arquitectura, realizarán con el sistema (figura 8).

Estructura de clases: según afirman Booch, Rumbaugh y Jacobson (2006), las clases son los bloques de construcción más importantes de cualquier sistema orientado a objetos. Las clases guardan una correspondencia con los objetos físicos y lógicos del dominio del problema; es decir,

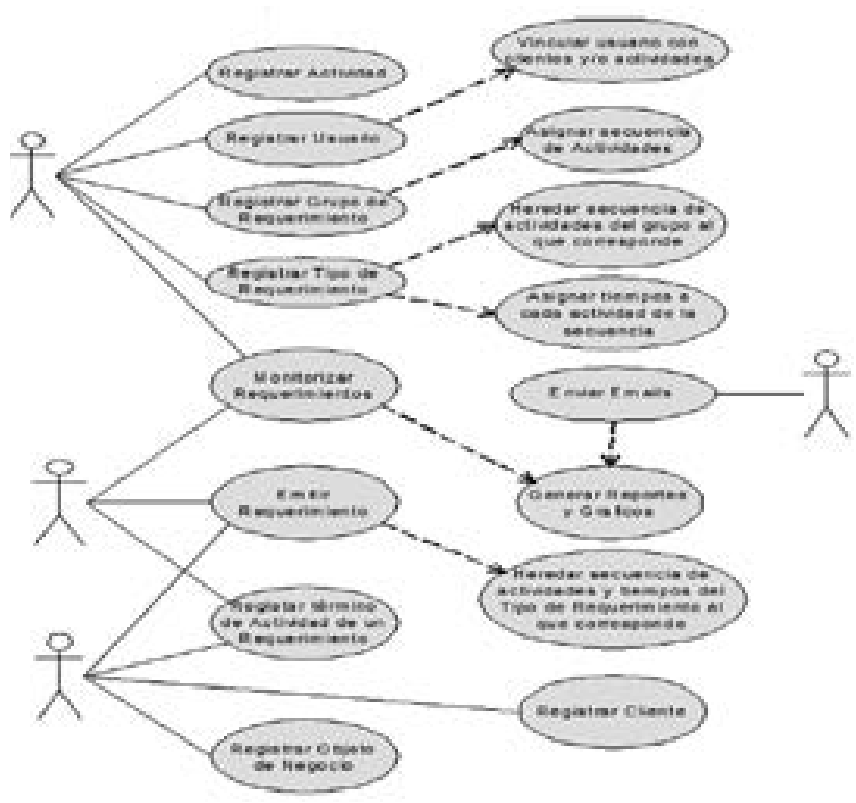

Fig. 8. Diagrama de casos de uso. constituyen las categorías en las que se agrupan los elementos principales del sistema.

Tomando este principio, se identificaron las clases relevantes y se construyó el diagrama de clases del sistema, que presenta todas las categorías de objetos y las relaciones entre ellos, lo que permite apreciar el sistema de nivel superior. La figura 9 presenta el diagrama de clases correspondiente.

Estructura de datos: para implementar las clases definidas, se desarrolló un modelo de datos relacional que presenta las entidades y relaciones necesarias para el prototipo.

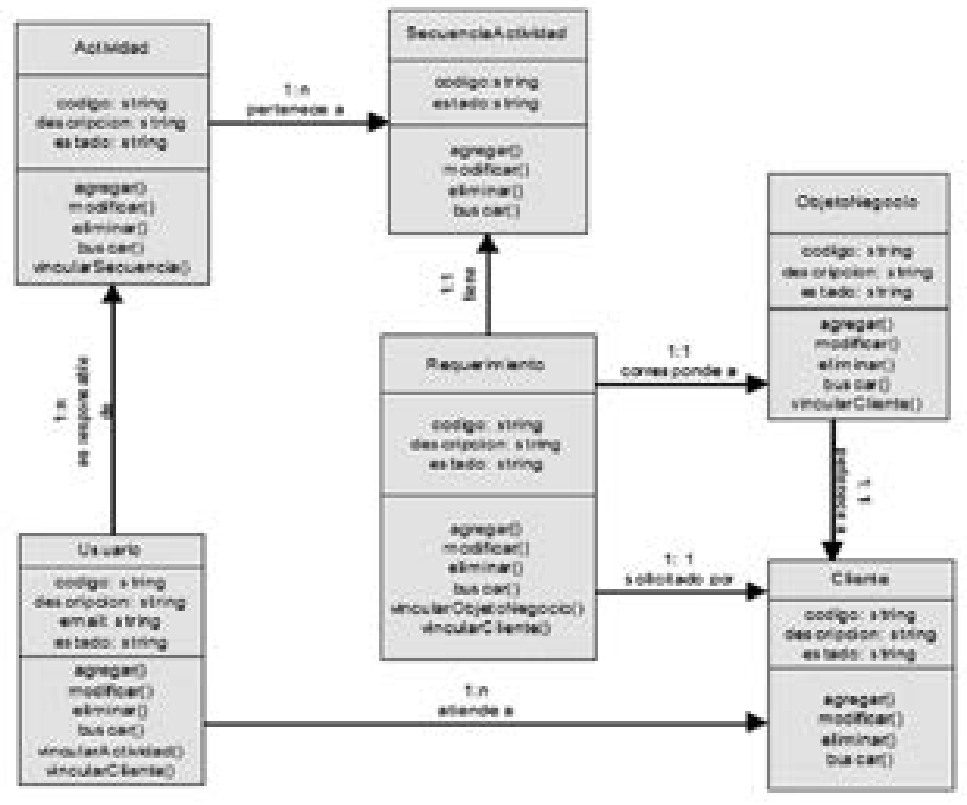

Fig. 9. Diagrama de clases del sistema. 
Prototipo del sistema: está conformado por el formulario para el registro de los tipos de requerimientos, el cual indica la secuencia de actividades y a qué clientes está vinculado. El formulario para el registro de usuarios permite vincularlos a los clientes y a las actividades. También se deben considerar los formularios para emitir requerimientos y descargar actividades realizadas y un formulario central para el monitoreo de todos los requerimientos, que permite mostrar, en un solo cuadro, el resumen de requerimientos pendientes por cliente y actividad. Asimismo, es posible apreciar los pendientes por cliente-actividad, así como el detalle por actividad (figura 10). Con el fin de demostrar la generalización, se aplicó el modelo ORA al otro 50\% de la muestra de requerimientos. Se pudieron modelar los 34 que restaban.

Se encontró que se mantuvieron las seis secuencias diferentes de actividades inicialmente encontradas con el análisis de la mitad de la muestra. Además, se hallaron ocho diferentes actividades para el modelado de todos los flujos de trabajo y cinco objetos de negocio.

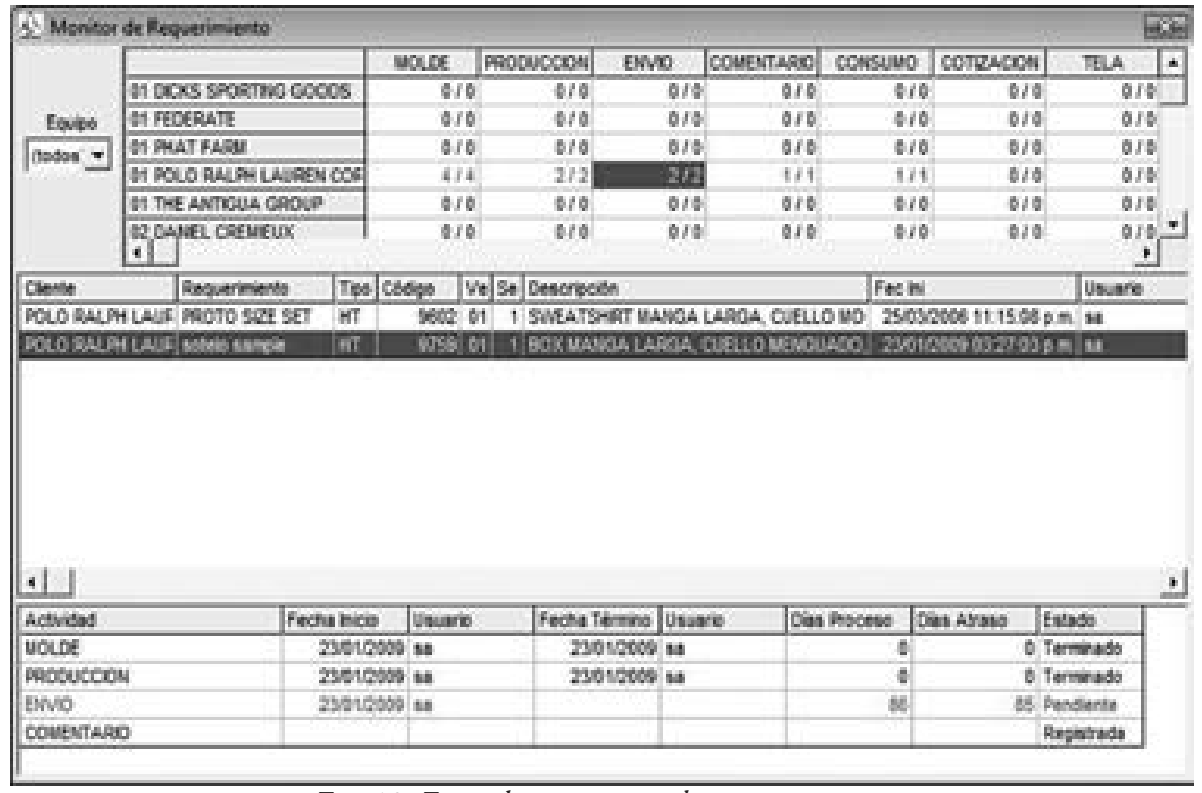

Fig. 10. Formulario monitor de requerimientos.

\section{CONCLUSIONES Y TRABAJO A FUTURO}

1. El modelo ORA (Objeto-Requerimiento-Actividad) es simple, pero eficaz para modelar los diferentes flujos de trabajo que las áreas de DDP de la Industria de la Confección deben atender.

2. La generalización del modelo se comprobó con el modelado de los 64 requerimientos que conformaron la muestra de datos.

3. La viabilidad se demostró con el prototipo de software que se desarrolló para implementar el modelo ORA.

4. Se comprobó la utilidad del modelo de estructura funcional de un WMS descrito por Muehlen (2004). El modelo ORA tomó como base dicha estructura.

5. Los conceptos de clase, herencia e instancia del diseńo orientado a objetos, fueron de suma importancia para generar la estructura dinámica del modelo ORA. Los tipos de flujo de trabajo para 
cada especie de requerimiento constituyeron las clases. Cada flujo de trabajo para una ocasión de un requerimiento constituyó la instancia de una clase determinada y heredó los atributos de dicha clase.

6. La implementación de sistemas de flujo de trabajo requiere una estrategia de integración que evite el doble registro en referencia a los objetos de negocio que generan los requerimientos y al registro de avance de las actividades del flujo. Este punto ofrece una oportunidad de extender la investigación.

\section{REFERENCIAS}

[1] Beltrán, J. (2004). Guía para una gestión basada en procesos. Instituto Andaluz de Tecnología.

[2] Bernal, C. (2006). Metodología de la investigación. Prentice Hall.

[3] Booch, G., Rumbaugh, J. y Jacobson, I. (2006). El lenguaje unificado de modelado. Addison Wesley.

[4] Borja, A. Delgadillo, G. (2004). Redes de petri extendidas para workflows y su aplicación en el diseño de una herramienta para el análisis de procesos. Bogotá: Departamento de Ingeniería Industrial, Universidad de los Andes.

[5] Garimella, M. y Williams, B. (2008). Introducción a BPM. Wiley Publishing, Inc.

[6] Hernández, R., Fernández, C. y Baptista, P. (2006). Metodología de la investigación. Mc Graw Hill.

[7] Muehlen, M. (2004). Workflow-based process Controling: Foundation, Design and Applications of Workflow-driven Process Information Systems. Alemania: s. e.

[8] Ogalla, F. (2006). Sistema de gestión: una guia práctica. Ediciones Díaz de Santos.

[9] Penadés, C. (2002) Una aproximación metodológica al desarrollo de flujos de trabajo. (Tesis de Doctorado). Departamento de sistemas informáticos y computación. Universidad Politécnica de Valencia, España.

[10] Petkov, E. y Haller, A. (2005). Aspects in Workflow Management. Digital Enterprise Research Institute, National University of Ireland.

[11] Sotelo, J. (2007). Workflow: Tecnología en Beneficio del Cliente. Punto y Plano, revista del Comité de Confecciones de la Sociedad Nacional de Industrias.

[12] Workflow Management Coalition Members. (1999). Terminology \& Glosary. Technical report WfMC-TC-1011. Recuperado de www.wfmc.org 